\title{
A Case Report and Review of Literature:
}

\section{Laing Early-onset Distal Myopathy Due to the MYH7 Mutation in an Iranian Family}

\author{
Shabnam Ghazanfari-Sarabi ${ }^{1} \odot$, Mostafa Rayati $^{2} \odot$, Mohammad Bagher Hashemi-Soteh $^{3^{*}} \odot$ \\ 1. Novin Genetics Diagnostic Laboratory, Sari, Iran. \\ 2. Department of Radiology, Faculty of Medical Sciences, Babol University of Medical Sciences, Babol, Sari, Iran. \\ 3. Immunogenetic Research Center, Molecular and Cell Biology Research Center, School of Medicine, Mazandaran University of Medical Sciences, Sari, Iran.
}

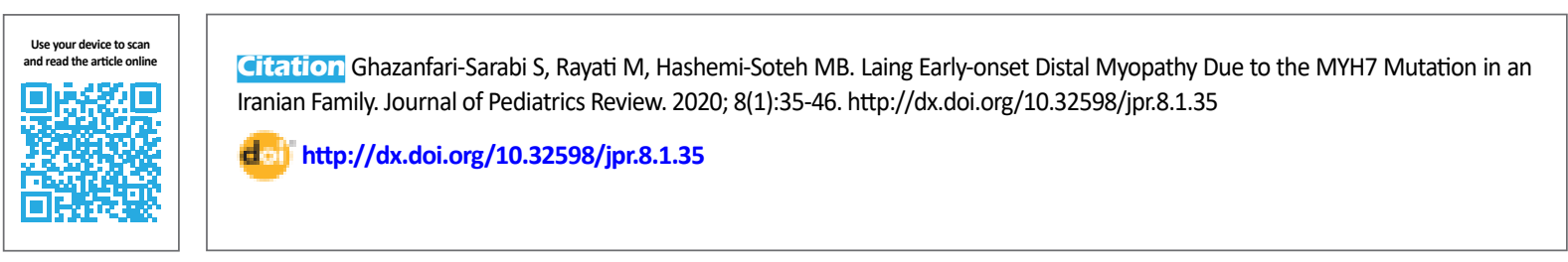

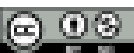

Article info:

Received: 10 Apr 2019

First Revision: 11 Jul 2019

Accepted: 23 Jul 2019

Published: 01 Jan 2020

\begin{abstract}
A B S T R A C T
Introduction: Laing early-onset distal myopathy is a disorder with autosomal dominant inheritance pattern caused by a mutation in the MYH7 gene that encodes the human $\beta$-myosin heavy chain. Most previous studies reported this disorder with mild symptoms involving foot and hand fingers extensors as its early-onset and neck flexors as late-onset symptoms. In this study, the previously reported cases suffered from the same mutation are reviewed, too.

Case Presentation: Our study describes pathological, clinical, imaging, and genetic findings in the first Iranian patient suffering from Laing distal myopathy. The subject is an 8-year-old boy with a moderate phenotype and upper and lower limbs involvement. He also showed a weakness in neck flexors, previously reported in similar cases at early ages. A genetic study was done using the whole exome sequencing method. Next generation sequencing findings revealed a c.48504852AGA deletion (p.k1617del) mutation in MYH7, which previously reported as the cause of Laing distal myopathy. This case is the first indication of Laing distal myopathy from Iran.

Conclusions: Presenting the first Iranian patient with an already known MYH7 mutation associated with Laing distal myopathy will prove the previously reported heterogeneity of this disorder's phenotype severity, morphological variation, and age of symptoms onset.
\end{abstract}

\section{Introduction}

ocated on chromosome 14, MYH7 gene encodes slow or $\beta$-myosin Heavy Chain (My$\mathrm{HCl}$ ) and is in tandem with the responsible gene for encoding $\alpha-\mathrm{MHC}$ (MYH6). MYH7 gene contains $22883 \mathrm{bp}$, including 41 exons. Except for two exons in 5 primes that are not being translated, 38 of them encode a protein, which consists of 1935 amino acids. Exons 3-21 are responsible for encoding the spherical head part embracing head and neck region and exons 22-40 encode the rod part of $\mathrm{MyHCl}$ involving the hinge and the light myosin chain (1). $\mathrm{MyHCl}$ is expressed in human type I striated muscle fibers. MYH7 mutations mainly cause myopathic cardiac disorders and skeletal myopathies, such

* Corresponding Author

Mohammad Bagher Hashemi-Soteh, PhD.

Address: Immunogenetic Research Center, Molecular and Cell Biology Research Center, School of Medicine, Mazandaran University of Medical Sciences, Sari, Iran.

Tel: +98 (911) 1164034

E-mail: hashemisoteh@mazums.ac.ir 
as distal myopathy and other kinds of skeletal muscle malformations that stem from thick filament aggregation in the sarcomeres.

Clinical symptoms of distal Myopathy-1 (MPD1), also known as Laing distal myopathy, range from scapuloperoneal phenotype and classical distal to axial and respiratory engagement, asymptomatic elevated serum Creatine Kinase (CK), known as hyperCKemia (limbgirdle) (2-4). MPD1 is identified by lower limbs distal deficiency with early juvenescence onset, selectively encompassing Tibialis Anterior (TA) muscle, and extensor halluces longus muscles, leading to hanging big toe and bilateral steppage. Weakness gradually progresses along with age to affect neck flexors and finger extensors $(2,5)$. Thus far, MYH7-related distal myopathy has been suggested as a rare disorder, and only a few cases have been reported across the globe $(1,5)$. Recent publications have considered MYH7 mutations as the fundamental element of a vast proportion of a broad skeletal myopathies spectrum $(2,3)$. Despite the clinical phenotype of MPD1 that suggests as rather homogeneous, considerable variability in onset age, the dispensation of weaknesses, and findings of muscle biopsy have been found even in patients who suffer from the same mutations $(2,3,5)$.

In spite of clinical findings that reveal similar results, biopsy findings include various features, such as endomysial fibrosis, type (I) fiber atrophy, fatty replacement, and myofiber degeneration. Moreover, mitochondrial dysfunction, including ragged red fibers, have been mentioned with rimmed vacuoles, especially in those presented with body myositis. (5).

Here we describe an Iranian family with an autosomal dominantly inherited pattern of symptomatic MPD1 harboring a known p.K1617del mutation in MYH7. This family remarkably widens the spectrum of clinicopathological aspects related to MPD1, manifesting the first case of MPD1 in Iran.

\section{Case Presentation}

An 11-year-old boy (subject III.1) presented at our outpatient clinic, complained of the overly flexible muscles, especially in his neck (Figure 1A). The affected patient could walk independently; showed generalized muscle atrophy of the upper and lower limbs, also severe proximal and distal muscles weakness in neck flexors (Figure $1 \mathrm{~A}$ and $1 \mathrm{~B}$ ). On examination, the severe weakness of intrinsic hands musculature on both sides was seen. Moreover, the patient showed a deficiency in the flexion and extension of the arms and elbow extensor muscle group. He presented with toe walking and foot drop since age 4 and developed a steppage gait after three to four months, which led him to use ankle-foot orthoses (Figure $1 \mathrm{C}$ ).

The patient underwent a cardiological examination to investigate systolic and diastolic ventricles function by 2D echocardiography, which indicated normal left ventricular systolic and diastolic functions. It has been previously mentioned that Electromyography (EMG) studies in peripheral neuropathy, shows merely a reduced recruitment pattern of motor units of normal appearance. EMG in subject III.1 illustrated a reduction in recruitment pattern for biceps, deltoid, extensor digitorum muscle, and gastrocnemius. High amplitude, long duration motor unit potentials with an average number of phases are related to chronic reinnervation. Practically, motor unit potentials are less than two $\mathrm{mV}$ in amplitude with a duration of ten to five ms with three to four phases.

In chronic partial denervation where intramuscular sprouting and reinnervation occur, amplitudes may be $10-20 \mathrm{mV}$ and durations as $20-30 \mathrm{~ms}$. Based on an increase in the amplitude of biceps, deltoid and extensor digitorum muscles on the right side and TA, Gastrocnemius (GCS), and quadriceps femoris muscles on both right and left sides, we concluded neurogenic pattern in this patient. In addition, fibrillation was seen in TA of both sides (1 Table). Family pedigree revealed the fact that other family members were also affected by muscular disorders (Figure 2A).

Subject II.3 was a 38-year-old woman with difficulty in walking stemming from ankle dorsiflexion, which she overcame it by wearing ankle-foot orthoses (Figure 1D). She presented toe walking and steppage gait by the age of seven. She also presented clinical features of ankle dorsiflexion, and typical hand extensor digitorum weakness, especially in her thumbs and fifth finger started in her 30s (Figure 1E). She gave birth to two sons (III.1 and III.2) (Tables 2 and 3), both presented with steppage gait by the age of three to four years. No cardiac involvement (echocardiogram) was detected in patient known as subject II.3.

Subject III.2 was a 9-year-old boy who developed a mild state of walking difficulties and ankle dorsiflexion (Figure 1F). EMG findings in this patient showed no Motor Unit Potentials (MUPs) in the quadriceps and TA but increased MUPs in extensor digitorum and biceps brachii muscles on both sides (Table 3). Moreover, reduced 
or discrete recruitment in this muscle except for extensor digitorum, which its recruitment was full, indicated the generalized neurogenic process (Table 1). No cardiac involvement was detected, too.

Subject II.2 was a 34-year-old man who suffered from the seizure, unsteadiness in walking, hand tremors, hand numbness, and bradykinesia. No cardiac involvement was detected in this patient. Furthermore, to specify the phenotype of the family, additional siblings subjected to examination genetically at our clinic. All members filled an informed consent form to cooperate in this study. The study protocol conforms to the ethical guidelines of the 1975 Declaration of Helsinki as reflected in a priori approval by the institution's human research committee.

Subject III.1 showed a persistent elevation of Creatine Phosphokinase enzyme level (CPK) in a moderate range of $276 \mathrm{IU} / \mathrm{L}$ (Table 2).
A

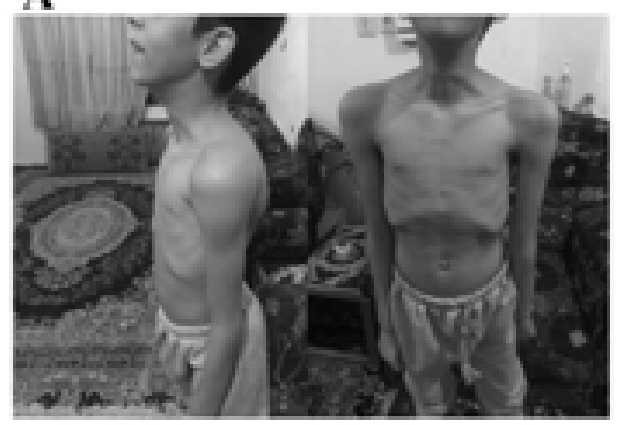

B

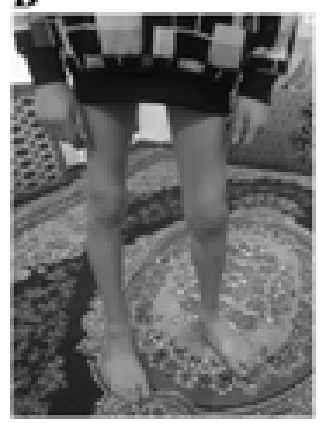

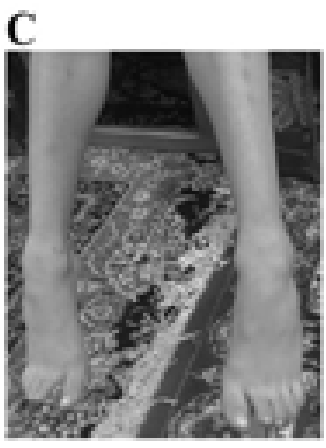

E

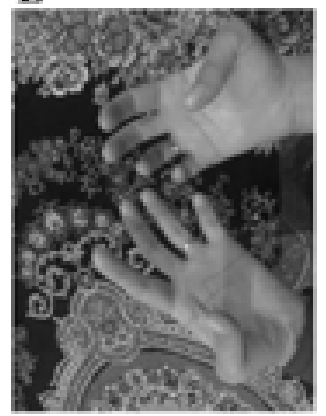

D

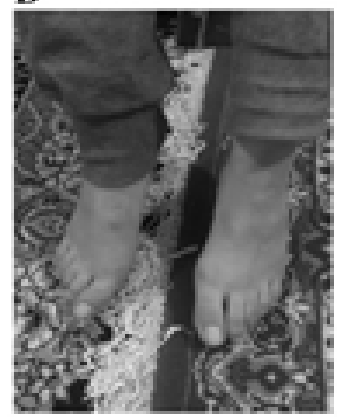

$\mathbf{F}$

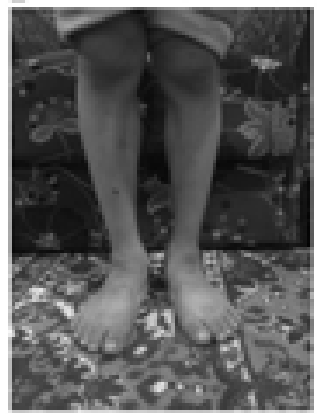

Figure 1. Photographs are presenting clinical features of the subjects III.1, III.2, and II.3

Journal of Pediatrics Review

A. Displaying bilateral quadriceps atrophy, bilateral atrophy of anterior compartment muscles in the lower legs, calves hypertrophy, and distal leg wasting in sub III.1; B. Featuring hanging big toe sign and bilateral foot drop; C. Subject III.1 showed neck flexor weakness result in difficulty in extending the neck; D. Hanging big toe sign and calves hypertrophy, especially in the left leg of subject III.2; E. Hanging big toe sign of subject II. 3; F. Finger extensor weakness, especially in the first and fifth fingers in the subject II.3 
A

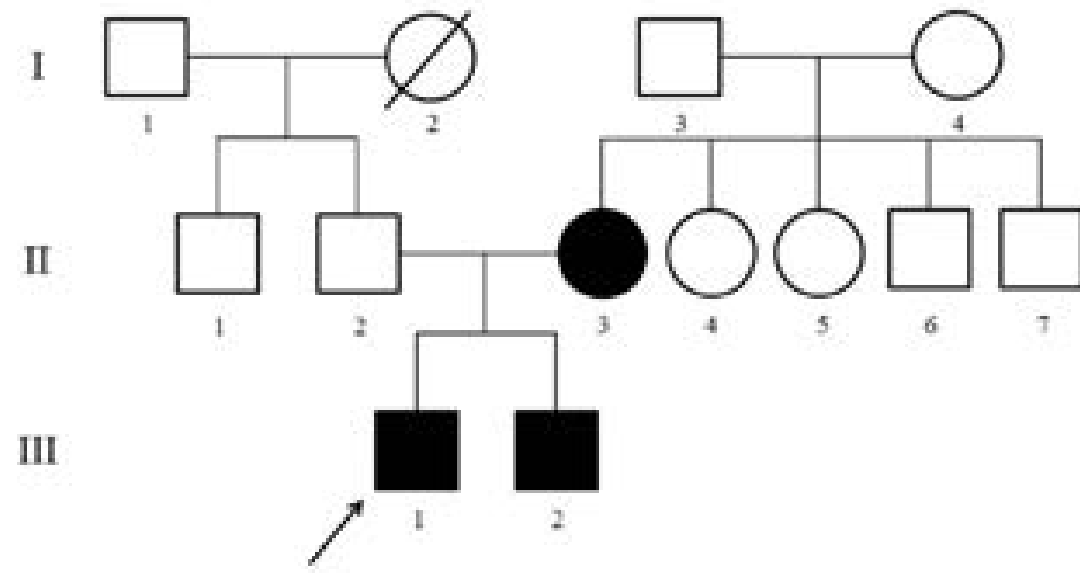

B

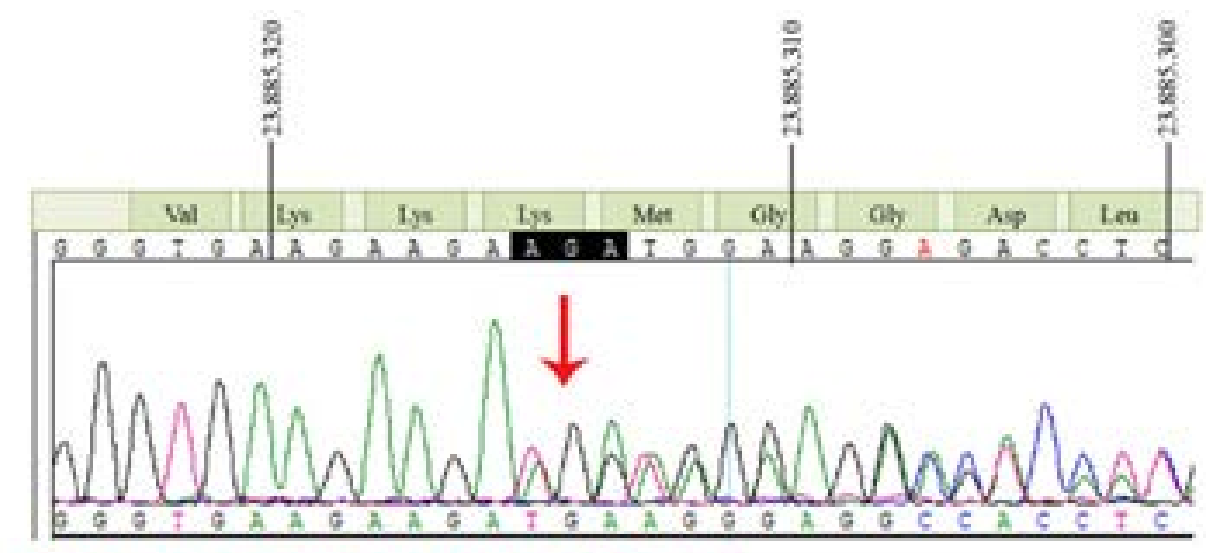

MYH7;NM_000257;c.4850_4852delAGA;-

Journal of Pediatrics Review

Figure 2. Pedigree of family and next generation sequencing result

A. Family tree and genetic features of the reported patient, bearing an MYH7 mutation from an Iranian family; $B$. Next generation sequencing findings followed by Sanger sequencing result of the MYH7 gene, shows 3 bp AGA deletion (c.4850-4852 del AGA) in exon 34, deleting the third lysine from a three-lysine-stretch (p.K1617del)

Genomic DNA from subject III.1 was obtained from peripheral blood based on the standard protocol. The DNA sample was sequenced by a high throughput Next Generation Sequencing (NGS) method using BGI genomics services (Beijing, China). We applied a targeted panel for muscular dystrophy, provided for analyzing the coding sequence of 79 genes responsible for muscular dystrophy. The findings revealed $\mathrm{MYH7}$ gene 3bp deletion (c.4850-4852delAGA) in exon 34, codon 32 , which deletes the third lysine from a three-lysinestretch (p.K1617del) originally investigated for autosomal dominant distal muscular atrophy (4). The mother started toe walking at three years and lost ambulation at age 27 . Her three daughters presented with early steppage gait and showed variable progression. Muscle biopsies were nonspecific showing myogenic lesions in the mother and lesions resembling neurogenic atrophy in the two siblings.

To identify the causative abnormality whole exome sequencing was performed in two affected girls and their unaffected father, unexpectedly revealing the $\mathrm{MYH7}$ mutation c.4849_4851delAAG (p.K1617del in the related protein (Figure 2). The result was verified by Sanger sequencing. This mutation is an already-described mutation resulting in MPD1 (OMIM \#160500) (6). Other family members, including parents and the affected brother, were also tested for this mutation using the Sanger sequencing method that verified the same heterozygous variant in the affected mother and brother, 
but not the father. There was no evidence available to show a mutation in other genes related to the myogenic or neurogenic pattern of symptoms. Due to these pieces of evidence and based on the previously reported studies, we conclude that MPD1 in our patients was associated with the MYH7 mutation.

\section{Discussion}

MPD1 possesses a well-defined and known natural history, according to the age of onset, usually from 4 to 35 year in most patients. Generally, the severity of the disease is rather mild and does not result in the loss of ambulation in advanced age (7). This study seeks to describe the first Iranian patient suffers from the MPD1 (OMIM \#160500).

By applying muscular dystrophy next generation sequencing panel (79 gene analysis), we described the previously known p.K1617del mutation in the MYH7 gene responsible for encoding the slow myosin heavy chain protein with dominant inheritance, initially examined for atypical distal muscular atrophy (4). The disap- pearance of one of the three successive lysines (AAG) is caused by p.K1617del. Meredith et al. assumed that it might happen through the same mechanism that altered the size of microsatellite allele previously described by Xu et al. $(6,8)$. Because the mutated residue is located in a three-repeat microsatellite, it probably plays a part in the mutation's reoccurrence during polymerase slippage (9).

The identified mutation has occurred in exon 34, which is related to the myosin tail. The tail of the myosin is the fundamental factor of the myosin dimerization and thick filament conformation. The deletion of one residue in myosin tail distorts the heptad-repeat quintessential to the coiled-coil formation (10). This mutation can remarkably alleviate the coiled-coil formation possibility in myosin tail (6). The mutation that causes Laing myopathy can inhibit the myosin binding to M-protein, myomesin, or titin. The myomesin and M-line proteins like M-protein are bound to the set of residues located on 1503-1671 (11). Mutation in our study patients was situated in aforementioned area (1503-1671 residues) that caused Laying myopathy, distorted the binding

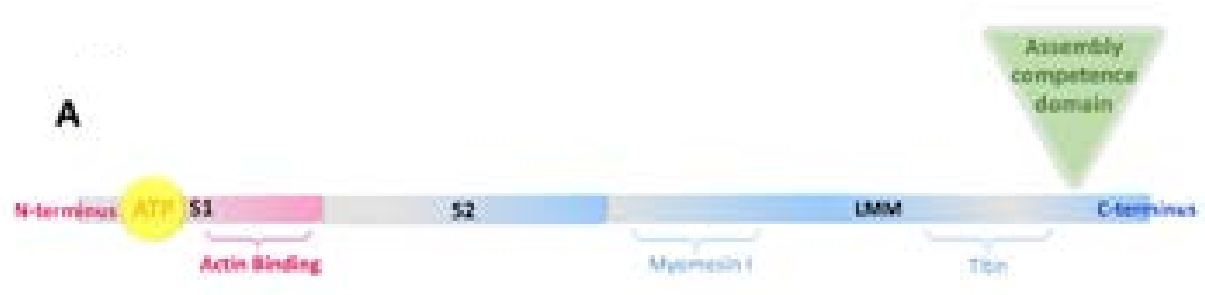

B

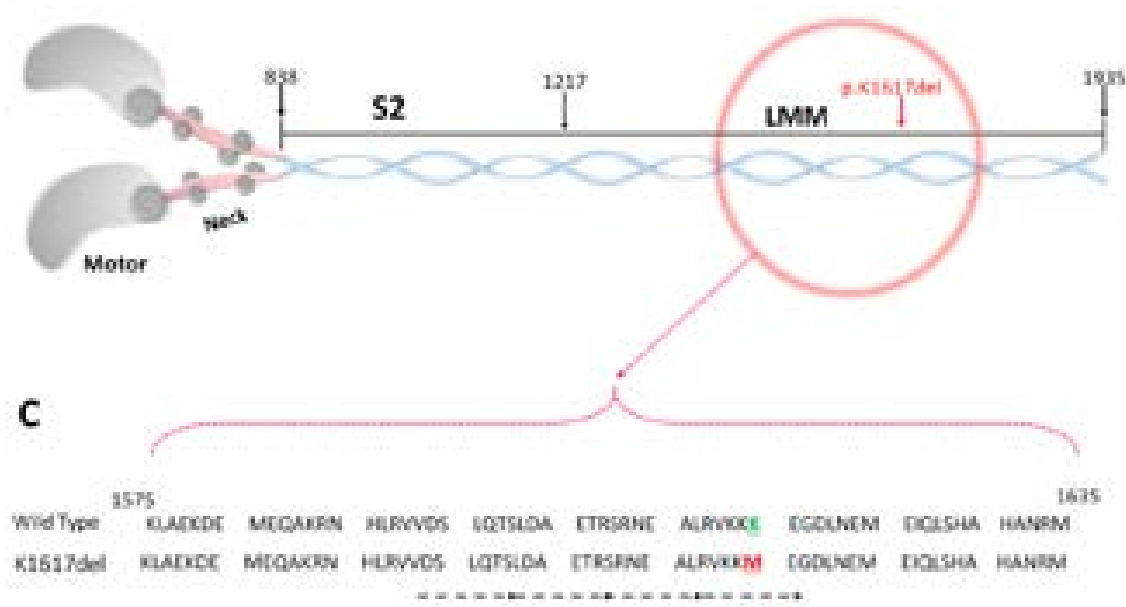

Journal of Pediatrics Review

Figure 3. Myosin structure and the location of the p.K1617del mutation

A. Schematic depiction of myosin protein, including its domains; B. Molecular model to show the position of our mutation along with LMM structure indicating by amino acid numbers; $\mathrm{C}$. A section of $\beta$-MHC representing the wild-type amino acid sequence versus the mutant amino acid sequence for $\mathrm{p} . \mathrm{K} 1617 \mathrm{del}$ 
site of myosin to M-proteins, whereas the MYH7 mutation, which caused cardiomyopathy (supplementary information in Figure 1) and Myosin Storage Myopathy (MSM) was located in the globular head of the myosin (actin-binding site) and very distal end of the myosin tail (exon 37-40), respectively (Figure 3; supplementary information in Figure 1) $(1,12)$.

To illustrate, in MPD1 similar to the other distal myopathies, the EDB (extensor digitorum brevis) muscles are spared, while the anterior leg compartment muscles face atrophy. This state has been claimed as the differences between neurogenic muscle atrophy and myopathies, which is entirely compatible with the clinical symptoms of our patients (13). So far, the p.k1617del in the MYH7 encodes the rod domain of myosin-7 associated with MPD1, reported in various patients (4). None of our patients have shown any symptoms of cardiomyopathy yet. As previously reported for other MPD1 arginine mutations, the mutation reported here does not express cardiac involvement, although $\beta$-myosin is expressed in both skeletal and cardiac muscles (4, 13-15).

Besides, MPD1 severity is usually mild and slowly progressive, which led affected patients to preserve the ambulation potential even at advanced ages $(2,6)$. However, two Italian pedigrees presented severe-moderate phenotype with the involvement of proximal and distal muscles, which caused the loss of their ambulation in the elderliness (3). Admittedly, the family history of our patient suggests a de novo mutation in the maternal pattern. Although the missense variant influencing residue 1617 has already been reported as the cause of MPD1, we are unable to firmly establish the mode of transmission in our case, as grandparents were reportedly healthy.

The reason for the several phenotypes caused by the same mutation in MYH7 is still unknown. Leading non-specific muscle pathology and an EMG neurogenic pattern to a recurrent misleading diagnosis of a distal neuropathy in MPD1 patients have been reported earlier (4). However, Muelas et al. suggested an unknown process of atypical motor unit reassembling as the cause of this phenomenon (2). Before the Italian patient whose muscle biopsy exhibited the myofibrillar myopathies-like and cytoplasmic factors, the significant findings of muscle biopsy included non-specific fiber type disproportion (3). Also, Komlósi, K. et al. have recently reported myopathic signal in muscle biopsy findings of a patient in early adulthood, which later on muscle biopsy illustrated the pattern similar to non-specific changes and neurogenic atrophy (4).

\section{Review of Literature}

Laing Distal Myopathy (LDM) has been described in the scientific literature, with several reported cases worldwide. In this study, five cases which so far reported with the same mutation in different patients are being reviewed (Table 1). Zimprich et al. reported muscular dystrophy with an early adult-onset in an Austrian family, which was inherited in an autosomal dominant trait (16). This disorder was characterized by a selective and slowly progressive weakness, initially in the anterior tibial compartment muscles, which in some cases over the time involved the sternocleidomastoids and the fingers long extensors. Other proximal and distal muscles were spared, and there was no sign of cardiac conduction defects indicated the specificity of the pathophysiological process for specific skeletal muscles. In these patients, the serum CK levels were slightly elevated.

In 2001, Voit et al. reported the clinical and neuropathological features of a German family with autosomal dominant distal myopathy and onset in the age of two to three years, whose haplotype segregation was compatible with the linkage to the LDM locus on the 14th chromosome (17). The initial manifestation of the muscle disease was associated with weakness and selective wasting of the anterior tibialis and extensor digitorum longus muscles. The disorder slowly progressed over the decades, and at later stages, abdominal muscles, neck flexor, and hand extensor were affected more than trunk muscles, hand flexor, and ankle flexor. Voit and colleagues addressed the question of how the peripheral and the central nervous system were being involved in LDM by documenting a hypomyelinating peripheral neuropathy in the sural nerve (17).

Two patients of a family in their study suffered from reversible numbness and paresthesia in their legs and arms followed by unusual postures. In addition, all patients of this family developed hands tremor and later a postural tremor. However, more observation is needed to confirm a neurogenic involvement in this disorder. CK levels were normal or slightly elevated, and the affected muscle groups always functioned to maintain the full range of motion in a joint where the maximum force production lies with the dorsal muscle group.

Komlo' si et al. used a whole exome sequencing approach to identify the p.K1617del mutation in the MYH7 gene associated distal myopathy with a somewhat distinct phenotype in a Hungarian Roma family with dominant inheritance pattern in 2014 (4). Although the clinical symptoms in this family resembled the distal my- 
Table 1. The literature related to the p.K1617del mutation

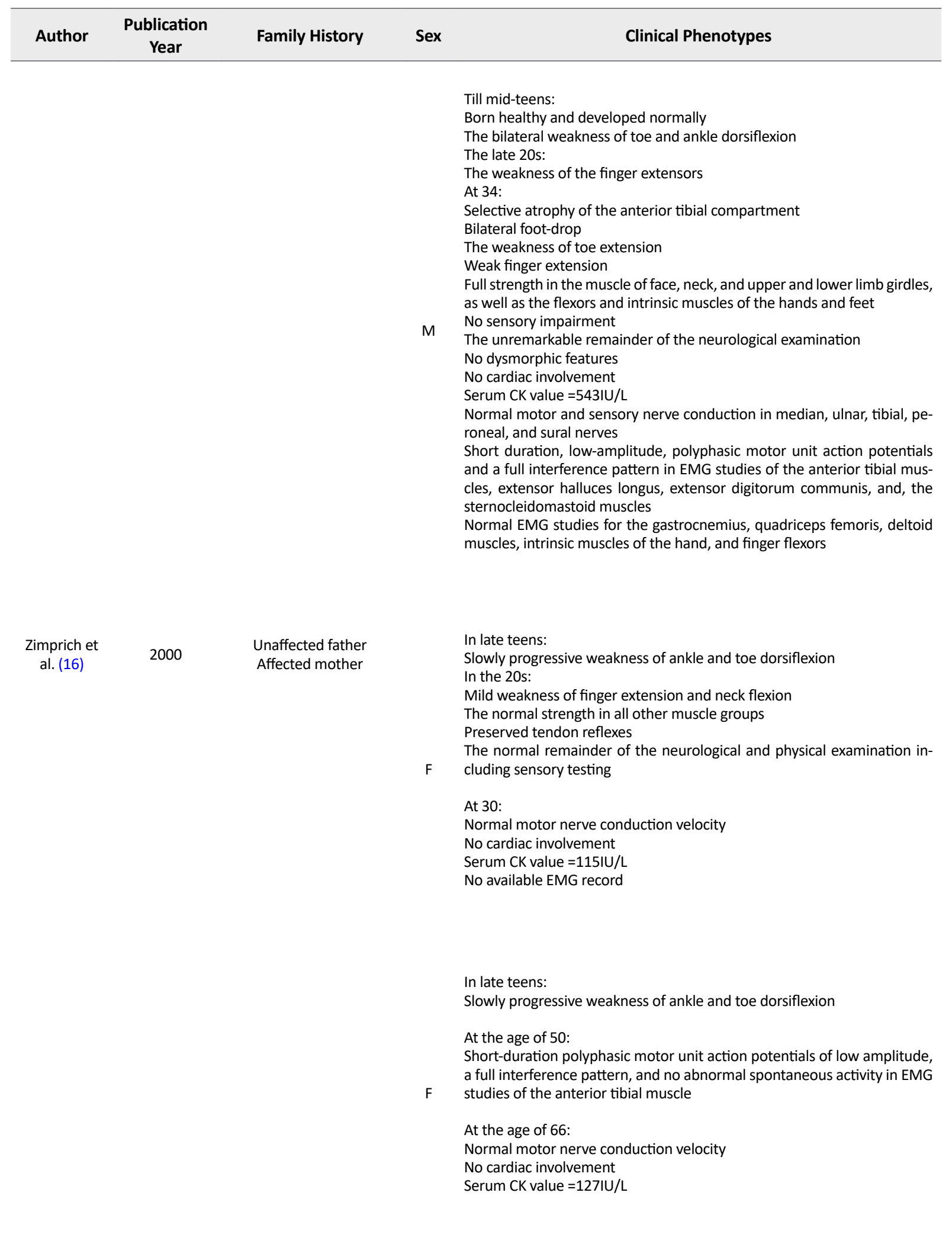




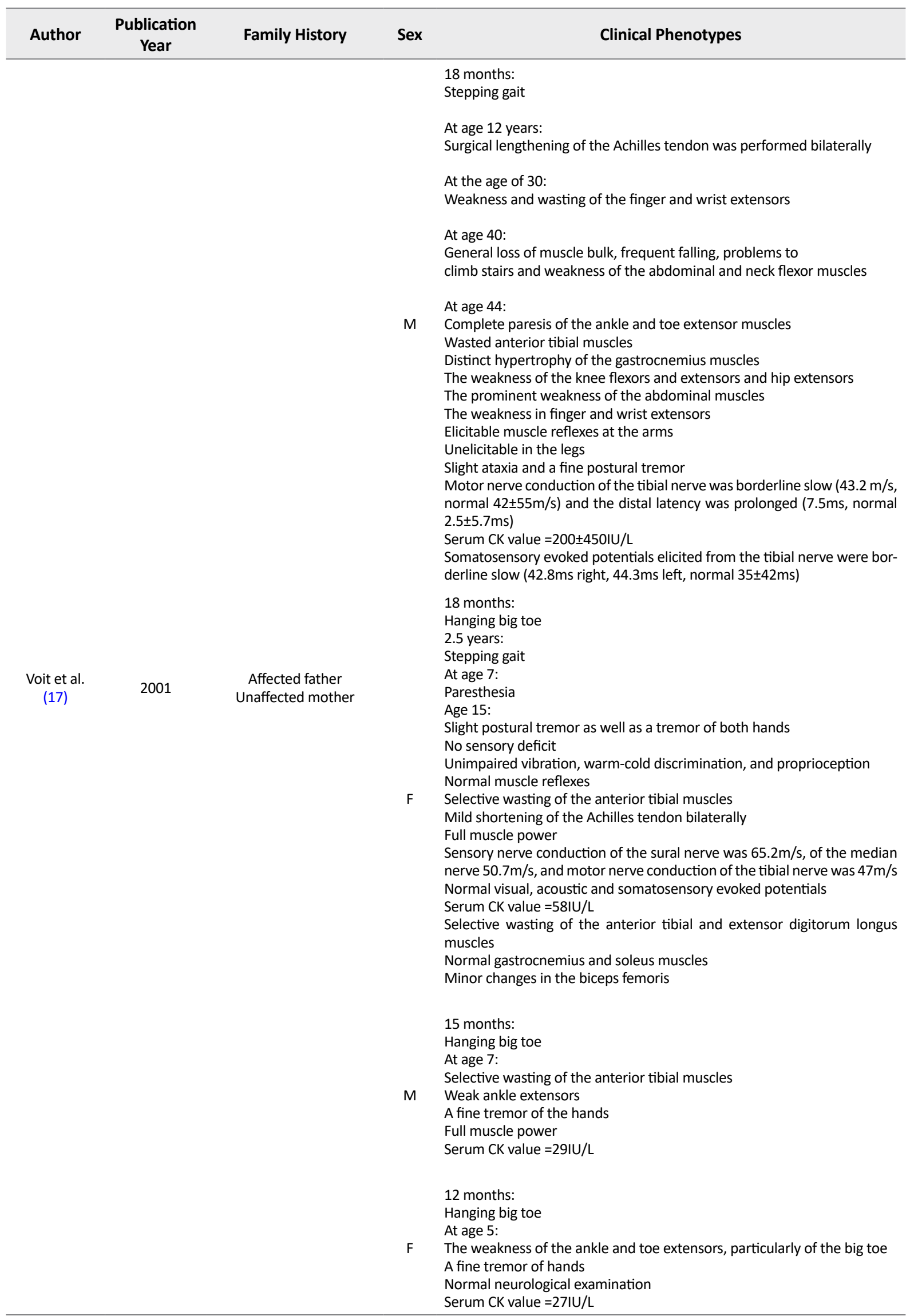




\begin{tabular}{|c|c|c|c|c|}
\hline Author & $\begin{array}{c}\text { Publication } \\
\text { Year }\end{array}$ & Family History & Sex & Clinical Phenotypes \\
\hline $\begin{array}{c}\text { Komlo' si et } \\
\text { al. (4) }\end{array}$ & 2014 & $\begin{array}{l}\text { Unaffected father } \\
\text { De novo mutation in } \\
\text { mother }\end{array}$ & $\mathrm{F}$ & $\begin{array}{l}\text { At age 2: } \\
\text { Toe walking } \\
\text { Pseudohypertrophy of the calves } \\
\text { Diminished deep tendon reflexes in the lower limbs } \\
\text { At age 4: } \\
\text { Steppage gait and pes excavatus on both feet } \\
\text { At age 6: } \\
\text { Achillotenotomy } \\
\text { Normal peroneal and tibial nerve } \\
\text { At age 7: } \\
\text { No abnormal muscle pattern } \\
\text { At age 8: } \\
\text { Slightly diminished muscle strength in the upper limbs and moderately } \\
\text { reduced strength in the distal lower limbs } \\
\text { Hanging toe and foot, mild paresis of the finger and wrist extensors } \\
\text { No affection in neck flexors }\end{array}$ \\
\hline & & & $\mathrm{F}$ & $\begin{array}{l}\text { At age 3: } \\
\text { Slight pseudohypertrophy of the calves } \\
\text { Slightly diminished deep tendon reflexes } \\
\text { At age 5: } \\
\text { Paresis in ankle dorsiflexors } \\
\text { Absence of all deep tendon reflexes of upper and lower limbs } \\
\text { Normal peroneal and tibial nerve study } \\
\text { At the age of 7: } \\
\text { Slight paresis of the finger and wrist extensors } \\
\text { Achillotenotomy } \\
\text { No affection in neck flexors }\end{array}$ \\
\hline $\begin{array}{l}\text { Oda et al. } \\
\text { (13) }\end{array}$ & 2015 & $\begin{array}{c}\text { Unaffected father } \\
\text { De novo mutation in } \\
\text { mother }\end{array}$ & $\begin{array}{c}\mathrm{M} \\
\text { and } \mathrm{F}\end{array}$ & $\begin{array}{l}\text { At birth: } \\
\text { Asymptomatic } \\
\text { 3-10 years old: } \\
\text { Onset of disease } \\
\text { Toe dorsiflexion difficulty and foot drop } \\
\text { Proximal limb weakness } \\
\text { Mild scapular winging, and or scoliosis } \\
\text { Calf muscle hypertrophy } \\
\text { Slowly progressive muscle weakness } \\
\text { Atrophy affecting the distal and proximal limbs and girdle } \\
\text { Decreased deep tendon reflexes } \\
\text { Positive Gower's sign } \\
\text { At the age of } 50 \text { : } \\
\text { The weakness of finger extensor and neck flexion } \\
\text { Relatively symmetric symptoms } \\
\text { No dysarthria, dysphagia, or facial muscle involvement } \\
\text { No high arched palate, tremors, sensory disturbances, contractures of } \\
\text { joints } \\
\text { No respiratory involvement } \\
\text { No cardiac involvement } \\
\text { Normal intelligence } \\
\text { Sclerosis in } 6 \text { patients } \\
\text { At the age of } 60-70 \text { : } \\
\text { Inability to walk without aids } \\
\text { Nerve conduction study revealed normal conduction velocity } \\
\text { Normal CK } \\
\text { Myogenic changes in EMG in two patient }\end{array}$ \\
\hline
\end{tabular}




\begin{tabular}{|c|c|c|c|c|}
\hline Author & $\begin{array}{l}\text { Publication } \\
\text { Year }\end{array}$ & Family History & Sex & Clinical Phenotypes \\
\hline \multirow{3}{*}{$\begin{array}{l}\text { Fiorillo et al. } \\
\text { (14) }\end{array}$} & \multirow{3}{*}{2016} & No family history & M & $\begin{array}{l}\text { At the age of } 4 \text { : } \\
\text { Dropped head due to the isolated weakness of neck extensors } \\
\text { Weakness in axial, distal upper and lower limbs } \\
\text { Respiratory involvement } \\
\text { Age of 8: } \\
\text { Hypertrophy of gastrocnemii } \\
\text { Non-compaction of the left ventricle } \\
\text { Recurrent pericarditis } \\
\text { Calves hypertrophy } \\
\text { The absence of rigid spine } \\
\text { Serum CK value }=7111 \mathrm{U} / \mathrm{L}\end{array}$ \\
\hline & & $\begin{array}{l}\text { Unaffected father } \\
\text { Affected but asymptom- } \\
\text { atic mother }\end{array}$ & $M$ & $\begin{array}{l}\text { Axial and distal weaknesses } \\
\text { At the } 20 \text { s: } \\
\text { No cardiac involvement } \\
\text { No respiratory involvement } \\
\text { Progressive weakness of axial muscle ending in a bent spine phenotype } \\
\text { Motor neuropathy predominantly affecting the lower limbs } \\
\text { Myopathic and neurogenic EMG } \\
\text { Serum CK value }=720 \text { IU/L }\end{array}$ \\
\hline & & $\begin{array}{l}\text { Positive family history } \\
\text { (Details are not available) }\end{array}$ & $F$ & $\begin{array}{l}\text { Tibialis anterior weakness } \\
\text { Weakness in proximal and distal upper and lower limb } \\
\text { Respiratory involvement } \\
\text { No cardiac involvement } \\
\text { Normal CK level } \\
\text { Myopathic EMG }\end{array}$ \\
\hline
\end{tabular}

Summary of Electromyography for subject III.1 and subject III.2

Journal of Pediatrics Review

Table 2. Cardiac examination results from patient III.1

\begin{tabular}{cccccc}
\hline Side & Muscle & Fibrillation & Amplitude & Duration & Recruitment \\
\hline RT & Biceps & - & Increased & Increased & Reduced \\
RT & Deltoid & - & Increased & Increased & Reduced \\
\hline RT & EDC & - & Increased & Increased & Reduced \\
RT \& LT & Tibialis Anterior & 1+/CRD & Increased & Increased & Discrete \\
RT \& LT & GCS§ & - & Increased & Increased & Reduced \\
\hline RT \& LT & Quadriceps & - & Increased & Increased & Discrete \\
\hline
\end{tabular}

†EDC: Extensor digitorum muscle; ¥CRD: Complex repetitive discharge; § GCS: Gastrocnemius

Journal of Pediatrics Review

Table 3. Cardiac examination results from patient III.2

\begin{tabular}{ccccccc}
\hline Side & Muscle & $\begin{array}{c}\text { Normal } \\
\text { MUPst }\end{array}$ & $\begin{array}{c}\text { Low } \\
\text { Amp }\end{array}$ & $\begin{array}{c}\text { High } \\
\text { Amp }\end{array}$ & Duration & Recruitment \\
\hline RT \& LT & Quadriceps & 0 & 0 & +3 & Long & Reduced \\
RT \& LT & $\begin{array}{c}\text { Tibialis anterior } \\
\text { RT }\end{array}$ & 0 & 0 & 0 & Long & Discrete \\
RT & $\begin{array}{c}\text { Extensor digitorum com- } \\
\text { munic } \\
\text { Biceps brachii }\end{array}$ & +3 & 0 & +1 & Normal & Full \\
\hline
\end{tabular}

+ Motor unit potentials

Journal of Pediatrics Review 
opathy, the electrophysiological and muscle pathology studies were consistent with the neurogenic pattern. Disregards from the same mutation, the progressive course of the disorder with a relatively early loss of ambulation and intrafamilial variability indicated the broad spectrum of myosinopathies phenotype. The members of this family showed a moderate finger extensor weakness during just two years, which illustrated the prompt progression of the disorder against other previously described myopathies.

There was not any sign of cardiac involvement; however, the writer believed that it might occur later on since the mechanism elucidating cardiac and or skeletal relationship was not understood. Disregards from the myopathic sign in the muscle biopsy in adulthood, biopsy for the mother of the family and two affected daughters later in life illustrated neurogenic atrophy pattern.

Oda et al. reported the clinical and genetic research of a large Chinese family with autosomal dominant myopathy through five generations (13). This study showed even a broader spectrum of the symptoms of the LDM caused by p.K1617del, since the onset of the disease varied from birth to 50 years of age in this family as well as the severity and distribution of muscle weakness. Cases in this family presented symptoms associated with impairment in truncal and proximal limbgirdle muscle, such as Gower's sign, scapular winging, and scoliosis in the early phase. The affected members of this family showed a faster progression of the disorder versus the typical cases of LDM and suffered from difficulty in walking in an advanced phase.

Fiorillo et al. study described a cohort of 21 Italian patients carried a total of 14 different mutations in MYH7 gene and displayed a broad spectrum of clinical presentations, which three of them suffered from the LDM caused by p.K1617del (14). One of these cases was diagnosed with longstanding motor neuropathy mostly affected the lower limb. Progressive weakness of axial muscle ending in a bent spine phenotype was developed in the 20s of this patient. However, since this patient carried two mutations, we could not lean on the disorder phenotype. The symptom of the disease for patients is summarized in Table 1. Fiorillo et al. confirmed that the site of mutation did not correlate with severity and could not anticipate phenotype (14). In this study, the patients indicated a variable phenotype, including a dropped head, and bent spine, which cannot be classified in previously described groups. This work confirms the complexity of this disorder by adding to the genotype-phenotype correlation of MYH7-related myopathies.

As described in this study, several cases with a p.K1617del mutation in the MYH7 gene have been reported that their clinical phenotypes varied greatly. The disease had an early adult-onset in an Austrian family reported by Zimprich et al., for instance, and in a German family had an onset in early childhood. With regards to the unknown reason for the wide variety of clinical symptoms for LDM associated with MYH7 mutation, Tasca et al. suggested that these symptoms might be affected by environmental modifiers or genetics (3). Moreover, it has been suggested that the ethnic differences might be associated with environmental factors and geographical variations of genetic backgrounds. Based on these additional clinical findings, our case suffered from a severe form of LDM with the same mutation, which could be the testimony on a broad clinical spectrum related to this deletion mutation. However, a broad disorder spectrum may lead to misdiagnosis with neurogenic atrophy, different congenital myopathies, and other neuromuscular conditions.

\section{Conclusions}

In this study, we described the first Iranian patient with a novel MYH7 mutation in our nation associated with MPD1 (OMIM \#160500), which adds remarkable heterogeneity to this disorder's phenotype severity spectrum, morphological variation, and age of symptoms' onset.

\section{Ethical Considerations}

\section{Compliance with ethical guidelines}

All ethical principles were observed in this research. The participants were informed about the purpose of the study; they were also assured about the confidentiality of their information, and if desired, the results of the research would be available to them.

\section{Funding}

This research did not receive any specific grant from funding agencies in the public, commercial, or not-forprofit sectors.

\section{Authors contribution's}

All authors contributed in preparing this article. 


\section{Conflicts of interest}

The authors declared no conflict of interest.

\section{References}

1. Tajsharghi $H$, Thornell $L E$, Lindberg $C$, Lindvall $B$, Henriksson KG, Oldfors A. Myosin storage myopathy associated with a heterozygous missense mutation in MYH7. Annals of Neurology. 2003; 54(4):494-500. [DOI:10.1002/ana.10693] [PMID]

2. Muelas N, Hackman P, Luque H, Garcés-Sánchez M, Azorín I, Suominen T, et al. MYH7 gene tail mutation causing myopathic profiles beyond Laing distal myopathy. Neurology. 2010; 75:732-41. [DOI:10.1212/WNL.0b013e3181eee4d5] [PMID]

3. Tasca G, Ricci E, Penttilä S, Monforte M, Giglio V, Ottaviani $\mathrm{P}$, et al. New phenotype and pathology features in MYH7related distal myopathy. Neuromuscul Disord. 2012; 22(7):640-7. [DOI:10.1016/j.nmd.2012.03.003] [PMID]

4. Komlósi K, Hadzsiev K, Garbes L, Martínez Carrera LA, Pál E, Sigursson JH, et al. Exome sequencing identifies Laing distal myopathy MYH7 mutation in a Roma family previously diagnosed with distal neuronopathy. Neuromuscul Disord 2014; 24(2):156-61. [DOI:10.1016/j.nmd.2013.10.010] [PMID]

5. Lamont PJ, Udd B, Mastaglia FL, de Visser M, Hedera P, Voit $\mathrm{T}$, et al. Laing early onset distal myopathy: Slow myosin defect with variable abnormalities on muscle biopsy. Journal of Neurology, Neurosurgery \& Psychiatry. 2006; 77(2):208-15. [DOI:10.1136/jnnn.2005.073825] [PMID] [PMCID]

6. Meredith C, Herrmann R, Parry C, Liyanage K, Dye DE, Durling $\mathrm{HJ}$, et al. Mutations in the slow skeletal muscle fiber myosin heavy chain gene (MYH7) cause laing early-onset distal myopathy (MPD1). The American Journal of Human Genetics. 2004; 75(4):703-8. [DOI:10.1086/424760] [PMID] [PMCID]

7. Oldfors A, Lamont PJ. Thick filament diseases. Advances in Experimental Medicine and Biology. 2008; 642:78-91. [DOI:10.1007/978-0-387-84847-1_7] [PMID]

8. Xu X, Peng $M$, Fang $Z, X u X$. The direction of microsatellite mutations is dependent upon allele length. Nature Genetics. 2000; 24(4):396-9. [DOI:10.1038/74238] [PMID]

9. Viguera E, Canceill D, Ehrlich SD. Replication slippage involves DNA polymerase pausing and dissociation. The EMBO Journal. 2001; 20(10):2587-95. [DOI:10.1093/emboj/20.10.2587] [PMID] [PMCID]

10. O'Neil KT, DeGrado WF. A thermodynamic scale for the helix-forming tendencies of the commonly occurring amino acids. Science. 1990; 250(4981):646-51. [DOI:10.1126/science.2237415] [PMID]
11. Obermann WM, van der Ven PF, Steiner F, Weber K, Fürst DO. Mapping of a myosin-binding domain and a regulatory phosphorylation site in M-protein, a structural protein of the sarcomeric $\mathrm{M}$ band. Molecular Biology of the Cell. 1998; 9(4):829-40. [DOI:10.1091/mbc.9.4.829] [PMID] [PMCID]

12. Blair E, Redwood C, de Jesus Oliveira M, Moolman-Smook $\mathrm{JC}$, Brink $\mathrm{P}$, Corfield VA, et al. Mutations of the light meromyosin domain of the beta-myosin heavy chain rod in hypertrophic cardiomyopathy. Circulation Research. 2002; 90(3):263-9. [DOI:10.1161/hh0302.104532] [PMID]

13. Oda $\mathrm{T}$, Xiong $\mathrm{H}$, Kobayashi $\mathrm{K}$, Wang $\mathrm{S}$, Satake $\mathrm{W}$, Jiao $\mathrm{H}$, et al. A de novo mutation of the MYH7 gene in a large Chinese family with autosomal dominant myopathy. Human Genome Variation. 2015; 2(15022):1-7. [DOI:10.1038/ hgv.2015.22] [PMID] [PMCID]

14. Fiorillo C, Astrea G, Savarese M, Cassandrini D, Brisca G, Trucco F, et al. MYH7-related myopathies: Clinical, histopathological and imaging findings in a cohort of Italian patients. Orphanet Journal of Rare Diseases. 2016; 11(1):91 [DOI:10.1186/s13023-016-0476-1] [PMID] [PMCID]

15. Lamont PJ, Wallefeld W, Hilton-Jones D, Udd B, Argov Z, Barboi $A C$, et al. Novel mutations widen the phenotypic spectrum of slow skeletal/ $\beta$-cardiac myosin (MYH 7) distal myopathy. Human Mutation. 2014; 35(7):868-79. [DOI:10.1002/humu.22553] [PMID] [PMCID]

16. Zimprich F, Djamshidian A, Hainfellner JA, Budka H, Zeitlhofer J. An autosomal dominant early adult-onset distal muscular dystrophy. Muscle \& Nerve. 2000; 23(12):1876-9. [DOI:10.1002/1097-4598(200012)23:123.0.CO;2-A]

17. Voit T, Kutz P, Leube B, Neuen-Jacob E, Schröder JM, Cavallotti D, et al. Autosomal dominant distal myopathy: Further evidence of a chromosome 14 locus. Neuromuscular Disorders. 2001; 11(1):11-9. [DOI:10.1016/S09608966(00)00158-9] 